\title{
Anomalies involving the space of couplings and the Zamolodchikov metric
}

\section{Yuji Tachikawa}

Kavli Institute for the Physics and Mathematics of the Universe, University of Tokyo, Kashiwa, Chiba 277-8583, Japan

E-mail: yuji.tachikawa@ipmu.jp

ABSTRACT: The anomaly polynomial of a theory can involve not only curvature two-forms of the flavor symmetry background but also two-forms on the space of coupling constants. As an example, we point out that there is a mixed anomaly between the R-symmetry and the topology of the space of exactly marginal couplings of class $\mathrm{S}$ theories. Using supersymmetry, we translate this anomaly to the Kähler class of the Zamolodchikov metric. We compare the result against a holographic computation in the large $N$ limit.

Keywords: Anomalies in Field and String Theories, Supersymmetry and Duality, AdSCFT Correspondence

ArXIV EPRINT: 1710.03934 


\section{Contents}

1 Introduction and summary 1

2 Field theoretical computations 2

2.1 The $4 \mathrm{~d}$ anomaly from the $6 \mathrm{~d}$ anomaly 2

2.2 Finding the Kähler potential 3

3 Holographic computations 4

\section{Introduction and summary}

The anomaly polynomial $\mathbb{A}_{D+2}$ of a $D$-dimensional quantum field theory encodes the anomalous variation of the phase of the partition function in the presence of the background fields in terms of the well-known descent procedure. We often think of $\mathbb{A}_{D+2}$ as a functional of the background gauge fields and of the background metric, but it is known from the early days of the study of anomalies that it can also depend on the topology of the space of couplings $[1-3] .^{1}$

One example can be constructed as follows. Take an arbitrary target space $\mathcal{M}$ and a $\mathrm{U}(N)$ gauge field $\mathcal{A}$ on it. Consider a $D$-dimensional free theory of a dynamical chiral fermion $\psi$ in the fundamental of $\mathrm{U}(N)$ coupled to a background scalar $\phi$ which is a map to $\mathcal{M}$ in the following manner: the fermion $\psi$ is minimally coupled to the pull-back under $\phi$ of the $\mathrm{U}(N)$ field $\mathcal{A}$. Of course the theory has the anomaly polynomial

$$
\mathbb{A}_{D+2}=\hat{A}(T X) \operatorname{tr} e^{i \phi^{*}(\mathcal{F})}
$$

where $X$ is the worldvolume of the theory and $\mathcal{F}$ is the curvature two-form on $\mathcal{M}$. When the scalar $\phi$ is considered dynamical, this is known under the name of the $\sigma$-model anomaly, and makes the theory ill-defined when not canceled. When $\phi$ is considered as a background field, this anomaly polynomial involves differential forms on the space $\mathcal{M}$ of couplings, and serves as one of the characteristic properties of the theory, just as the ordinary 't Hooft anomalies do.

To readers who found the example above rather artificial, let us provide a more meaningful case. In [5] Gaiotto introduced a large class of $4 \mathrm{~d} \mathcal{N}=2$ theories obtained by compactifying a $6 \mathrm{~d} \mathcal{N}=(2,0)$ theory on a Riemann surface $C$, possibly decorated with punctures. Here let us consider a simple case where $C$ is of genus $g$ without any puncture. This construction gives rise to a family of $4 \mathrm{~d} \mathcal{N}=2$ superconformal field theories (SCFTs), now

\footnotetext{
${ }^{1}$ Strictly speaking in these papers the scalars were considered dynamical. But before 't Hooft [4] the gauge fields in the anomalies were also mostly considered dynamical. In this sense the anomaly involving the space of couplings is known from the early days.
} 
known as class $\mathrm{S}$ theories, whose space of exactly marginal couplings is the moduli space $\mathcal{M}_{g}$ of the genus- $g$ Riemann surfaces. We show below that this theory has a hithertounappreciated term in the anomaly polynomial of the form

$$
\mathbb{A}_{6} \supset c_{2}(R)\left[\frac{\omega}{2 \pi}\right]
$$

where $c_{2}(R)$ is the second Chern class of the background $\mathrm{SU}(2)_{R}$ gauge field and $[\omega]$ is a degree-2 cohomology class on $\mathcal{M}_{g}$, which can be determined from the known anomaly polynomial of the $6 \mathrm{~d} \mathcal{N}=(2,0)$ theory [6-8].

Now, a mixed anomaly between the Weyl transformation and the Kähler transformation of the space of exactly marginal couplings of general $4 \mathrm{~d} \mathcal{N}=2$ SCFTs was described in $[9-11] .{ }^{2}$ When one reads their derivation carefully, one finds that their analysis already implies an anomaly of the form (1.2) above, with an added bonus that $[\omega]$ is proportional to the cohomology class $\left[\omega^{\mathrm{Z}}\right]$ of the Kähler form $\omega^{\mathrm{Z}}$ of the Zamolodchikov metric. Turning the logic around, this means that we can easily fix $\left[\omega^{Z}\right]$ in terms of the anomaly polynomial of the $6 \mathrm{~d} \mathcal{N}=(2,0)$ theory.

Finally, we note that the Zamolodchikov metric and therefore $\omega^{Z}$ is computable in the large $N$ limit by means of the AdS/CFT correspondence [13] using the holographic dual of the class $\mathrm{S}$ theories $[14,15]$. This is known to be proportional to the standard Weil-Petersson metric on $\mathcal{M}_{g},{ }^{3}$ but the precise proportionality coefficient has not been computed to the author's knowledge. We will show below that the class of the Kähler form computed holographically is compatible with the computation from the anomaly as above, using a classic mathematical result by Wolpert $[16,17]$.

The rest of the note is devoted to implement the computations outlined above: in section 2 we compute $\mathbb{A}_{6}$ of the class $\mathrm{S}$ theory from the anomaly of the $6 \mathrm{~d} \mathcal{N}=(2,0)$ theory and then use it to compute the Kähler class $\left[\omega^{\mathrm{Z}}\right]$ of the Zamolodchikov metric. Then in section 3 we compute the Zamolodchikov metric using holography, determine the proportionality coefficient with respect to the standard Weil-Petersson metric, and compare it against the result in section 2 .

\section{Field theoretical computations}

\subsection{The 4d anomaly from the $6 \mathrm{~d}$ anomaly}

We start from the anomaly polynomial [6-8] of the $6 \mathrm{~d} \mathcal{N}=(2,0)$ theory of type $G=$ $A_{n-1}, D_{n}, E_{6,7,8}$ :

$$
\mathbb{A}_{8}=\frac{h_{G}^{\vee} d_{G}}{24} p_{2}(N Y)+\frac{r_{G}}{48}\left(p_{2}(N Y)-p_{2}(T Y)+\frac{1}{4}\left(p_{1}(N Y)-p_{1}(T Y)\right)^{2}\right) .
$$

\footnotetext{
${ }^{2}$ There they conjectured that the Kähler potential would be globally well defined but this was answered negatively in a recent paper [12]. This latter paper actually gave the impetus of the investigation which led to this short note.

${ }^{3}$ The author does not know who originally noticed this; he forgot from whom he first learned the fact. This is surely a common knowledge among those who study class $\mathrm{S}$ theories using AdS/CFT.
} 
Here $Y$ is the worldvolume of the theory, $T Y$ is its tangent bundle, $N Y$ is the $\mathrm{SO}(5) \mathrm{R}$ symmetry bundle, and $p_{1}, p_{2}$ are the Pontryagin classes; $h_{G}^{\vee}, d_{G}$ and $r_{G}$ are the dual Coxeter number, the dimension and the rank of the Lie algebra of type $G$. We use the convention that the anomaly polynomial gives the $(D+2)$-dimensional phase $\exp \left(\int_{Y_{D+2}} 2 \pi i \mathbb{A}_{D+2}\right)$.

The $\mathcal{N}=2$ class $\mathrm{S}$ theory of our interest is obtained by compactifying the $6 \mathrm{~d}$ theory on a Riemann surface $C$ of genus $g$ without any punctures so that we introduce a nonzero curvature to the subgroup $\mathrm{SO}(2) \subset \mathrm{SO}(5)$ of the R-symmery which cancels the curvature of $C$. This means that the Chern roots of $N Y$ is $\pm 2 \alpha$ and $\pm t$ where $\pm \alpha$ are the Chern roots of the $\mathrm{SU}(2)_{R}$ background field and $t$ is the $c_{1}$ of the tangent bundle of $C$. We note that $c_{2}(R)=-\alpha^{2}$.

Using $p_{1}=\sum_{i} \lambda_{i}^{2}$ and $p_{2}=\sum_{i<j} \lambda_{i}^{2} \lambda_{j}^{2}$ when the Chern roots are $\pm \lambda_{i}$, we easily get

$$
\mathbb{A}_{6} \supset-\left(\frac{h_{G}^{\vee} d_{G}}{6}+\frac{r_{G}}{12}\right) c_{2}(R) \int_{C} t^{2} .
$$

In the last factor, $t$ is considered as the $c_{1}$ of the relative tangent bundle of $C$ over $\mathcal{M}_{g}$ (i.e. the tangent bundle of the universal bundle $\mathcal{U}$ where $C \hookrightarrow \mathcal{U} \rightarrow \mathcal{M}_{g}$ minus the pull back of the tangent bundle of $\mathcal{M}_{g}$ ). Then $t^{2}$ is a 4 -form on $\mathcal{U}$, and we obtain a 2 -form on $\mathcal{M}_{g}$ by integrating over the fiber $C$.

\subsection{Finding the Kähler potential}

Suppose now that a given $4 \mathrm{~d} \mathcal{N}=2$ SCFT has a space of exactly marginal couplings parameterized by $\mathcal{M}$ with local complex coordinates $\tau^{I}$. We normalize the corresponding exactly marginal operators so that they enter in the deformation of the Lagrangian as

$$
\frac{1}{\pi^{2}} \int d^{4} x\left(\tau^{I} \mathcal{O}_{I}+\bar{\tau}^{\bar{J}} \overline{\mathcal{O}}_{\bar{J}}\right)
$$

following the convention of [9-11]. We then define the Zamolodchikov metric $g_{I \bar{J}}^{\mathrm{Z}}$ by the formula

$$
\left\langle\mathcal{O}_{I}(x) \overline{\mathcal{O}}_{\bar{J}}(0)\right\rangle=\frac{g_{I \bar{J}}^{\mathrm{Z}}}{x^{8}}
$$

This is known to be Kähler: $g_{I \bar{J}}^{\mathrm{Z}}=\partial_{I} \bar{\partial}_{\bar{J}} K$.

In [11] the authors identified that the Kähler transformation $K \mapsto K+F+\bar{F}$ needs to be accompanied by a shift of the contact terms

$$
S \mapsto S+\frac{1}{192 \pi^{2}} \int d^{4} x d^{4} \theta \mathcal{E} \frac{F}{2}\left(\Xi-W^{\alpha \beta} W_{\alpha \beta}\right)+c . c,
$$

where we used the $\mathcal{N}=2$ supergravity superfields as used in [11]. Using the component expansion given in (5.5) of [18], we see that this shift contains the terms of the form

$$
S \mapsto S-\frac{1}{192 \pi^{2}} \int d^{4} x F \frac{1}{4} R(V) \underset{a b j}{i} \tilde{R}(V)_{i}^{a b j}+\cdots
$$

where $R(V)_{a b_{j}}^{i}$ is the background gauge field strength of the $\mathrm{SU}(2)_{R}$ symmetry. Recalling that the second Chern class is given by

$$
c_{2}(R)=\frac{d^{4} x}{16 \pi^{2}} R(V)_{a b j}^{i} \tilde{R}(V)_{i}^{a b j}
$$


we see that $K \mapsto K+F+\bar{F}$ is accompanied by

$$
S \mapsto S-\int \frac{F-\bar{F}}{48} c_{2}(R)+\cdots .
$$

The Kähler form of the Zamolodchikov metric is

$$
\omega^{\mathrm{Z}}=\partial \bar{\partial} K=d A \quad \text { where } \quad A=\frac{1}{2}(\partial-\bar{\partial}) K .
$$

Therefore, $K \mapsto K+F+\bar{F}$ does

$$
A \mapsto A+\frac{1}{2}(\partial F-\bar{\partial} \bar{F})=A+d\left(\frac{F-\bar{F}}{2}\right) .
$$

This means that the shift of the contact term (2.8) is exactly the gauge variation one obtains from the anomaly polynomial

$$
\mathbb{A}_{6} \supset-\frac{c_{2}(R)}{24}\left[\frac{\omega^{\mathrm{Z}}}{2 \pi}\right] .
$$

Let us specialize to the case of the class $\mathrm{S}$ theory on a genus- $g$ Riemann surface. Choosing the type to be $A_{N-1}$ and taking the large $N$ limit, we find that our results (2.2) and (2.11) imply

$$
\frac{\omega^{\mathrm{Z}}}{2 \pi} \sim 4 N^{3} \int_{C} t^{2}
$$

as cohomology classes.

\section{Holographic computations}

Weil-Petersson metric. First let us recall the Weil-Petersson metric on the moduli space $\mathcal{M}_{g}$ of the genus- $g$ Riemann surface. Given Beltrami differentials $\mu_{I}$ on a Riemann surface $C$, we define the Hermitean structure on them by

$$
h_{I \bar{J}}^{\mathrm{WP}}=\int_{C} \mu_{I} \bar{\mu}_{\bar{J}} d A
$$

where $d A$ is the area form of curvature -1 , so that $\int_{C} d A=4 \pi(g-1)$. Then the Kähler form of the Weil-Petersson metric is given by

$$
\omega^{\mathrm{WP}}=g_{I \bar{J}}^{\mathrm{WP}} d \tau^{I} d \bar{\tau}^{\bar{J}}=\frac{1}{2} h_{I \bar{J}}^{\mathrm{WP}} d \tau^{I} d \bar{\tau}^{\bar{J}} .
$$

It is a classic mathematical result by Wolpert $[16,17]$ that the relation

$$
\frac{\omega^{\mathrm{WP}}}{2 \pi^{2}}=\int_{C} t^{2}
$$

holds as differential forms, not just as cohomology classes. 
Maldacena-Nuñez solution. The holographic dual of the class $\mathrm{S}$ theory of type $A_{N-1}$ on a genus- $g$ Riemann surface is given in $[14,15]$. The $11 \mathrm{~d}$ metric is of the form

$$
\begin{aligned}
d s_{11}^{2}=\left(\pi N \ell_{p}^{3}\right)^{2 / 3} W^{1 / 3}[ & 2 d s_{A d S_{5}}^{2}+d s_{H_{2} / \Gamma}^{2} \\
& +d \theta^{2}+W^{-1} \cos ^{2} \theta\left(d \psi^{2}+\sin ^{2} \psi d \phi^{2}+2 \sin ^{2} \theta(d \chi+A)^{2}\right],
\end{aligned}
$$

where $d s_{A d S_{5}}^{2}$ is the $A d S_{5}$ metric of unit radius, $d s_{H_{2} / \Gamma}^{2}$ is the metric on the Riemann surface $C$ represented as a quotient of the Poincaré disk of curvature radius 1 by a discrete group $\Gamma$, the coordinates $\theta, \psi, \phi$ and $\chi$ parameterize the internal space which is topologically of the form $S^{4}$, and $W:=1+\cos ^{2} \theta$. Our convention is that the $11 \mathrm{~d}$ action is

$$
S_{11} \supset \frac{1}{16 \pi G_{N}^{(11)}} \int d^{11} x \sqrt{\mathrm{g} R}, \quad G_{N}^{(11)}=16 \pi^{7} \ell_{p}^{9} .
$$

The point is that the deformation of $C=H / \Gamma$ is parameterized by $\mathcal{M}_{g}$, the moduli space of genus- $g$ Riemann surfaces. The space $\mathcal{M}_{g}$ appears as the target space of the massless scalars in five dimensional supergravity, which in turn can be identified with the space of exactly marginal couplings of the dual SCFT.

Reduction to five dimensions. Let us proceed with our computation. Integrating over the four-sphere part, we have

$$
d s_{7}^{2}=\left(\pi N \ell_{p}^{3}\right)^{2 / 3}\left(2 d s_{A d S_{5}}^{2}+d s_{H_{2}}^{2}\right)
$$

with the $7 \mathrm{~d}$ action

$$
S_{7} \supset \frac{1}{16 \pi G_{N}^{(7)}} \int d^{7} x \sqrt{\mathrm{gR}}+\cdots
$$

where

$$
\frac{1}{G_{N}^{(7)}}=\frac{1}{G_{N}^{(11)}}\left(\pi N \ell_{p}^{3}\right)^{2} \frac{8}{3} \pi^{2} \sqrt{2}
$$

We now reduce it further to five dimensions, including the deformation of the Riemann surface. In general, under a small deformation $\mathrm{g} \mapsto \mathrm{g}+\mathrm{h}$, we have

$$
\mathrm{R} \mapsto \mathrm{R}+\frac{1}{4} \nabla_{\mu} \mathrm{h}_{\nu \rho} \nabla^{\mu} \mathrm{h}^{\nu \rho}+\cdots
$$

Now note that the Beltrami differential $\mu:=\tau^{I} \mu_{\bar{z}}^{z} I$ deforms the internal metric as

$$
|d z|^{2} \mapsto|d z+\mu d \bar{z}|^{2}
$$

which means that

$$
\mathrm{g} \mapsto \mathrm{g}+\mathrm{h}, \quad \mathrm{h}:=2(\mu+\bar{\mu})
$$

where the indices are appropriately placed. This means that upon reduction to $5 \mathrm{~d}$ one finds

$$
S_{5} \supset \frac{4 \pi(g-1)}{16 \pi G_{N}^{(7)}} \int d^{5} x \sqrt{\mathrm{g} R}+\frac{2 h_{I \bar{J}}^{\mathrm{WP}}}{16 \pi G_{N}^{(7)}} \int d^{5} x \sqrt{\mathrm{g}}{ }^{\mu \nu} \partial_{\mu} \tau^{I} \partial_{\nu} \bar{\tau}^{\bar{J}}
$$


Translation to the dual SCFT. At this point, we can use the formula [19] for the central charge $a \sim c$ to compute

$$
c=\frac{\pi R_{A d S_{5}}^{3}}{8 G_{N}^{(5)}}=\frac{N^{3}}{3}(g-1)
$$

which reproduces the standard result.

We are more interested in the Zamolodchikov metric on $\mathcal{M}_{g}$, for which we use the formula for the two-point function under AdS/CFT given in [20]. The formula says that given the action

$$
S_{5} \supset \frac{\eta}{2} \int d^{5} x \sqrt{\mathrm{g}} \mathrm{g}^{\mu \nu} \partial_{\mu} \phi \partial_{\nu} \phi
$$

for a real scalar $\phi$ in five dimensions, the corresponding operator has the two-point function

$$
\langle\underline{\mathcal{O}}(x) \underline{\mathcal{O}}(0)\rangle=\eta R_{A d S_{5}}^{3} \frac{24}{\pi^{2}} \frac{1}{x^{8}},
$$

with the caveat that the deformation is introduced via the coupling

$$
S \mapsto S+\int d^{4} x \phi \underline{\mathcal{O}}
$$

without an additional factor of $\pi^{2}$ in the denominator as in (2.3).

Carefully collecting all the factors, one finds

$$
g_{I \bar{J}}^{\mathrm{Z}}=\frac{1}{16 \pi G_{N}^{(7)}} R_{A d S_{5}}^{3} \frac{24}{\pi^{2}} 2 h_{I \bar{J}}^{\mathrm{WP}}\left(\pi^{2}\right)^{2}=4 N^{3} \frac{g_{I \bar{J}}^{\mathrm{WP}}}{\pi}
$$

meaning that

$$
\frac{\omega^{\mathrm{Z}}}{2 \pi} \sim 4 N^{3} \frac{\omega^{\mathrm{WP}}}{2 \pi^{2}}=4 N^{3} \int_{C} t^{2}
$$

in the large $N$ limit. This is consistent with the result (2.12) we obtained from the consideration of the anomaly in the last section.

\section{Acknowledgments}

The author thanks D. R. Morrison for discussions, and T. Maxfield for pointing out a minor error in (2.2) in an older version of the manuscript. The author is partially supported in part byJSPS KAKENHI Grant-in-Aid (Wakate-A), No.17H04837 and JSPS KAKENHI Grantin-Aid (Kiban-S), No.16H06335, and also supported in part by WPI Initiative, MEXT, Japan at IPMU, the University of Tokyo.

Open Access. This article is distributed under the terms of the Creative Commons Attribution License (CC-BY 4.0), which permits any use, distribution and reproduction in any medium, provided the original author(s) and source are credited. 


\section{References}

[1] G.W. Moore and P.C. Nelson, Anomalies in Nonlinear Sigma Models, Phys. Rev. Lett. 53 (1984) 1519 [INSPIRE].

[2] G.W. Moore and P.C. Nelson, The etiology of sigma model anomalies, Commun. Math. Phys. 100 (1985) 83 [INSPIRE].

[3] A. Manohar, G.W. Moore and P.C. Nelson, A comment on sigma model anomalies, Phys. Lett. B 152 (1985) 68 [inSPIRE].

[4] G. 't Hooft, Naturalness, Chiral Symmetry, and Spontaneous Chiral Symmetry Breaking, NATO Sci. Ser. B 59 (1980) 135.

[5] D. Gaiotto, $\mathcal{N}=2$ dualities, JHEP 08 (2012) 034 [arXiv:0904.2715] [INSPIRE].

[6] J.A. Harvey, R. Minasian and G.W. Moore, NonAbelian tensor multiplet anomalies, JHEP 09 (1998) 004 [hep-th/9808060] [INSPIRE].

[7] K.A. Intriligator, Anomaly matching and a Hopf-Wess-Zumino term in $6 d, N=(2,0)$ field theories, Nucl. Phys. B 581 (2000) 257 [hep-th/0001205] [INSPIRE].

[8] P. Yi, Anomaly of (2,0) theories, Phys. Rev. D 64 (2001) 106006 [hep-th/0106165] [INSPIRE].

[9] E. Gerchkovitz, J. Gomis and Z. Komargodski, Sphere Partition Functions and the Zamolodchikov Metric, JHEP 11 (2014) 001 [arXiv:1405.7271] [INSPIRE].

[10] J. Gomis and N. Ishtiaque, Kähler potential and ambiguities in $4 d \mathcal{N}=2$ SCFTs, JHEP 04 (2015) 169 [arXiv:1409.5325] [INSPIRE].

[11] J. Gomis, P.-S. Hsin, Z. Komargodski, A. Schwimmer, N. Seiberg and S. Theisen, Anomalies, Conformal Manifolds and Spheres, JHEP 03 (2016) 022 [arXiv:1509.08511] [INSPIRE].

[12] R. Donagi and D.R. Morrison, Conformal field theories and compact curves in moduli spaces,

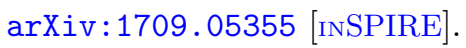

[13] J.M. Maldacena, The large-N limit of superconformal field theories and supergravity, Int. J. Theor. Phys. 38 (1999) 1113 [hep-th/9711200] [INSPIRE].

[14] J.M. Maldacena and C. Núñez, Supergravity description of field theories on curved manifolds and a no go theorem, Int. J. Mod. Phys. A 16 (2001) 822 [hep-th/0007018] [InSPIRE].

[15] D. Gaiotto and J. Maldacena, The gravity duals of $\mathcal{N}=2$ superconformal field theories, JHEP 10 (2012) 189 [arXiv:0904.4466] [INSPIRE].

[16] S. Wolpert, On the homology of the moduli space of stable curves, Ann. Math. 118 (1983) 491.

[17] S.A. Wolpert, Chern forms and the Riemann tensor for the moduli space of curves, Invent. Math. 85 (1986) 119.

[18] D. Butter, B. de Wit, S.M. Kuzenko and I. Lodato, New higher-derivative invariants in $\mathcal{N}=2$ supergravity and the Gauss-Bonnet term, JHEP 12 (2013) 062 [arXiv:1307.6546] [INSPIRE].

[19] M. Henningson and K. Skenderis, The holographic Weyl anomaly, JHEP 07 (1998) 023 [hep-th/9806087] [INSPIRE].

[20] D.Z. Freedman, S.D. Mathur, A. Matusis and L. Rastelli, Correlation functions in the $C F T_{d} / A d S_{d+1}$ correspondence, Nucl. Phys. B 546 (1999) 96 [hep-th/9804058] [INSPIRE]. 